\section{Estratificación de riesgo de muerte a mediano plazo de pacientes evaluados por enfermedad coronaria con SPECT de perfusión miocárdica bajo efecto de dipiridamol}

\author{
LUIS ALARCÓN ${ }^{1}$, MATÍAS RAU ${ }^{1, a}$, HERNÁN PRAT ${ }^{2}$, \\ RENÉ FERNÁNDEZ ${ }^{1, b}$, JAIME CARMONA ${ }^{1, b}$, JUAN P. ZHINDON ${ }^{1, b}$, \\ JAVIERA GONZÁLEZ ${ }^{1, b}$, TERESA MASSARDO ${ }^{1}$
}

\section{Predictive value of dipyridamole myocardial perfusion tomography on survival}

Background: Dipyridamole (DIP) is the most commonly employed pharmacological stressor for myocardial perfusion tomography (SPECT) in patients unable to reach an adequate work load. Aim: To assess the predictive capacity of DIP SPECT on survival. Material and Methods: We included 985 adults aged $66 \pm 11$ years ( $45 \%$ women) with rest and DIP-SPECT. The main indications for the procedure were coronary artery disease (CAD) screening in $66 \%$ and known CAD in 33\%. Participants were followed up for a median of 65 months (interquartile range 54 to 86 months). During the follow up, 261 deaths were recorded and $98 \%$ had a specified cause in their death certificate. Results: Myocardial SPECT was abnormal in 44\% of participants. Transient ischemic defects were observed in 34\%, fixed defects concordant with infarction in 27\% and post-stress systolic dysfunction in 23\%. Twenty five percent of deaths were attributable to cardiac or ischemic cause and $22 \%$ to cancer. In a bivariate analysis, the hazard ratio (HR) of death of any cause was lower in females and higher in the presence of $C A D$. The multivariate analysis showed that being older than 46 years increased the HR of death of any cause. In a bivariate analysis, the $H R$ for cardiac death was higher when the myocardial SPECT showed ischemia, necrosis or left ventricular dilation. In the multivariate analysis, post-stress left ventricular systolic function was associated with a lower risk of cardiac death. Conclusions: An abnormal myocardial SPECT, perfusion abnormalities, left ventricular systolic function or dilation are independent predictors of cardiac death in these participants.

(Rev Med Chile 2018; 146: 831-839)

Key words: Dipyridamole; Prognosis; Tomography, Emission-Computed, Single-Photon.

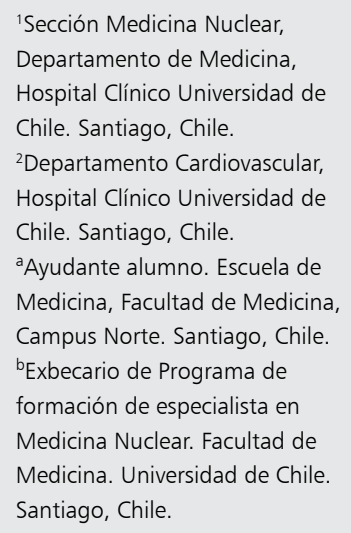

'Sección Medicina Nuclear, Departamento de Medicina, Hospital Clínico Universidad de Chile. Santiago, Chile.

${ }^{2}$ Departamento Cardiovascular, Hospital Clínico Universidad de Chile. Santiago, Chile.

${ }^{a}$ Ayudante alumno. Escuela de Medicina, Facultad de Medicina, Campus Norte. Santiago, Chile.

${ }^{\text {b}}$ Exbecario de Programa de

formación de especialista en Medicina Nuclear. Facultad de Medicina. Universidad de Chile. Santiago, Chile.

Los autores declaran no tener conflictos de interés.

Trabajo no recibió

financiamiento.

Recibido el 24 de mayo de 2018, aceptado el 24 de julio de 2018.

Correspondencia a:

Teresa Massardo Vega Sección Medicina Nuclear, Departamento de Medicina Hospital Clínico Universidad de Chile.

Santos Dumont 999 1-E, Independencia. Santiago, Chile. tmassardo@hcuch.cl

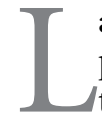

a enfermedad coronaria (EC) es uno de los problemas de salud pública más importantes, tanto a nivel internacional como nacional. En Estados Unidos de Norteamérica, se estima que 1 de 3 adultos tienen alguna forma de enfermedad cardiovascular ${ }^{1}$. En Chile, la EC es la principal causa de muerte específica, la que también se asocia a importante morbilidad secundaria a sus complicaciones. La tasa de mortalidad en el año 2009 por esta causa fue $32,1 / 100.000$ habitantes $^{2}$. 
Entre los principales estudios no invasivos para diagnosticar y estratificar riesgo en pacientes con EC conocida o sospechada está la tomografía por emisión de fotón único (SPECT) miocárdico, que permite determinar la presencia de isquemia, necrosis y tejido viable, dependiendo del tipo de defecto de perfusión y de la función ventricular ${ }^{3,4}$.

Las imágenes de perfusión miocárdica isotópicas (IPM) se realizan principalmente con tecnecio-99m (Tc99m), el que presenta menor tasa de exposición a radiación que el talio-201. Para detectar isquemia se requiere provocación, idealmente, con estrés en ejercicio en plataforma. Para el estrés farmacológico se utilizan vasodilatadores mediante infusión continua, adenosina y dipiridamol (DIP) o en bolo, regadenoson. El DIP tiene menor costo, pero su vida media es más larga, aunque sus efectos adversos pueden bloquearse con aminofilina. Los vasodilatadores inducen hiperemia miocárdica mediada por receptores de adenosina A2A, independiente de la demanda miocárdica de oxígeno, incrementando el flujo coronario 4 a 5 veces en vasos sanos; el DIP actúa aumentando niveles tisulares de adenosina, previniendo la recaptura intracelular y la deaminación de esta; la acción es inhibida por xantinas mediante bloqueo del receptor ${ }^{5-7}$. Otros marcadores no relacionados a perfusión, obtenidas en las IPM, de alto riesgo, incluyen alteraciones del electrocardiograma (ECG), anormalidad de motilidad de las paredes ventriculares inducidas por estrés, disminución de la fracción de eyección del ventrículo izquierdo (FEVI) postestrés $\geq 5 \%$, FEVI disminuida en ambas fases, dilatación isquémica transitoria del ventrículo izquierdo, aumento de captación del ventrículo derecho o aumento de actividad pulmonar por sobrecarga izquierda ${ }^{1,8}$.

La sensibilidad de las IPM varían desde $82 \%$ a $88 \%$ para ejercicio y $88 \%$ a $91 \%$ para estrés farmacológico y la especificidad de $70 \%$ a $88 \%$ y $75 \%$ a $90 \%$, respectivamente ${ }^{1}$. Sin embargo, en un metaanálisis reciente, al comparar con angiografía coronaria con medición de reserva de flujo fraccional, se encontró que el SPECT miocárdico tenía sensibilidad de $61 \%$, con especificidad de $84 \%{ }^{9}$; otra revisión mostró valores de $70 \%$ y $78 \%$ al analizar por paciente y de $57 \%$ y $75 \%$, respectivamente, por vasos comprometidos ${ }^{10}$.

La indicación de reemplazo del ejercicio por el estrés farmacológico está bien establecida en los consensos de criterios apropiados de uso de radionúclidos para perfusión miocárdica ${ }^{11,12}$. Por otra parte, es reconocido el gran valor pronóstico de la IPM, sobre todo el predictivo negativo, en que el riesgo de eventos cardiovasculares mayores (muerte de origen cardíaco o infarto del miocardio) es menor a $1 \%$ en un período de 2 a 3 años, incluso en pacientes con $\mathrm{EC}^{13}$. Sin embargo, existen grupos de alto riesgo clínico, como los diabéticos, en quienes los eventos anuales aumentan hasta $2-3 \%$, especialmente si presentan otros factores de riesgo cardiovascular (FRCV) $)^{14}$; así como aquellos que realizan estrés farmacológico, que se asocian a tasas anuales de $1-2 \%$, en oposición a $<1 \%$ en estudios normales con ejercicio ${ }^{15}$ por las condiciones basales inherentes a la indicación, que impiden la realización de una prueba adecuada y presentan peor sobrevida que aquellos con indicación de ejercicio, incluso sin considerar sus FRCV ${ }^{16,17}$.

El objetivo del presente estudio fue caracterizar nuestra población local, que efectúa IPM bajo efecto de estrés farmacológico con DIP y evaluar sobrevida a mediano plazo.

\section{Material y Métodos}

\section{Diseño del estudio}

Se incluyeron todos los estudios con DIP indicados en adultos, por diversas causas, realizados entre los años 2007 y 2012 con seguimiento posterior. Se registraron los datos demográficos, indicaciones y FRCV.

\section{Protocolo de estrés farmacológico y de adquisición, procesamiento e interpretación del SPECT}

La decisión de realizar estudio con estrés farmacológico estuvo basada en el requerimiento del médico tratante, pero la elección fue modificada por cardiólogo a cargo de la prueba electrocardiográfica. Se usó DIP en dosis estándar de $0,56 \mathrm{mg} / \mathrm{kg}$ en la mayoría y en algunos $0,84 \mathrm{mg} / \mathrm{kg}$. Se administró aminofilina intravenosa, como antagonista directo, en casos de reacciones adversas menores como cefalea, mareos, etc. Se entregó indicación de suspensión de xantinas (té, café, otro), al menos $12 \mathrm{~h}$ previas al estudio, pues disminuyen la sensibilidad por antagonismo directo ${ }^{7,18}$. Además, para comparar, a todos se les efectuó SPECT en reposo. La actividad administrada fue la habitual dependiendo del tipo de protocolo de 1 o 2 días. Se 
gatilló el SPECT con 8 cuadros en gammacámara Siemens E-CAM dual. La adquisición fue 45 a 60 minutos posterior a DIP. Si había defecto de perfusión, se agregaba nitritos sublinguales para evaluar eventual viabilidad. El estudio fue interpretado de forma visual y semicuantitativa con modelo de 17 segmentos del software QGS y QPS Cedars ${ }^{\circledR 13}$. La presencia de isquemia se identificó como defecto de perfusión en estrés reversible en reposo; los defectos fijos se consideraron necrosis (excluyendo variantes y atenuación de tejidos blandos) y viables si presentaban actividad mayor de 50\% del máximo de la pared. La función sistólica izquierda se determinó midiendo FEVI, cuyo valor normal es $\geq 45 \%$ con QGS Cedars ${ }^{\circledR}$. Se evaluó la calidad del gatillado con histograma de longitud de latidos.

\section{Seguimiento}

Los pacientes se siguieron con mediana de 65 meses (rango intercuartil de 54 a 86 meses); con mínimo de 0,15 y máximo de 107 meses registrando todas las causas de muerte, basados en registro civil nacional, separando origen cardiovascular u otro.

\section{Análisis estadístico}

Se realizó análisis bi y multivariado para cálculo de factores de riesgo para mortalidad global y de causa cardíaca, mediante regresión de Cox expresándose como Hazard Ratio (HR). Se efectuaron curvas de sobrevida, las que se compararon utilizando la prueba de Long-Rank y se graficaron las curvas de Kaplan-Meier.

Este estudio retrospectivo contó con aprobación del Comité Ético Científico del Hospital Clínico de la Universidad de Chile.

\section{Resultados}

De un total de 1.000 estudios analizados con SPECT DIP, se registraron 985 casos de adultos con fases de estrés y reposo. Ver Tabla 1 con sus características generales; $34 \%$ de los casos presentaban EC conocida y durante el seguimiento se consignaron $261(27 \%)$ muertes totales ocurridas durante 0,15 a 101 meses post-SPECT.

En la Tabla 2, se incluyeron los resultados principales obtenidos de los estudios de SPECT miocárdicos. El 44\% presentó alguna anormalidad, en 34\% hubo defectos transitorios; 265 pacientes

\section{Tabla 1. Características de los pacientes sometidos a SPECT miocárdico con estrés farmacológico}

\begin{tabular}{|lcr|}
\hline & & $\%$ \\
\hline Total de casos & 985 & 100 \\
Mujeres & 439 & 45 \\
\hline $\begin{array}{l}\text { Edad en años, promedio } \pm \text { DS } \\
\text { (rango) }\end{array}$ & $66 \pm 11$ & \\
Pacientes mayores de 65 años & $(32-95)$ & \\
\hline Enfermedad coronaria conocida & 576 & 58 \\
\hline Historia de infarto de miocardio & 333 & 34 \\
\hline Historia de revascularización coronaria & 117 & 12 \\
\hline Síntomas anginosos & 141 & 14 \\
\hline Hipertensión arterial & 353 & 36 \\
\hline Diabetes mellitus & 203 & 21 \\
\hline Enfermedad arterial oclusiva & 76 & 8 \\
\hline Enfermedad renal crónica & 29 & 3 \\
\hline Preoperatorio no cardíaco & 221 & 22 \\
\hline Bloqueo completo de rama izquierda & 22 & 2 \\
\hline Usuarios de marcapasos & 20 & 2 \\
\hline Seguimiento en meses, promedio \pm & $66 \pm 25$ & \\
\hline DS (rango) & $(0,15-107)$ & \\
\hline Muertes totales & 261 & 27 \\
\hline Muertes cardíacas & 66 & 7 \\
\hline
\end{tabular}

Tabla 2. Resultados de los SPECT de perfusión miocárdica con dipiridamol de un total de 985 estudios con 437 casos con alguna anormalidad

\begin{tabular}{|c|c|c|}
\hline & Alteración & $\%$ \\
\hline \multirow[t]{8}{*}{ Parámetro } & $\begin{array}{l}\text { Defecto de perfusión transitorio } \\
\text { isquémico }\end{array}$ & 34 \\
\hline & Defecto de perfusión fijo & 27 \\
\hline & $\begin{array}{l}\text { Función anormal del ventrículo } \\
\text { izquierdo }\end{array}$ & 29 \\
\hline & FEVI en reposo $<45 \%$ & 22 \\
\hline & FEVI en postestrés < $45 \%$ & 23 \\
\hline & Caída de FEVI $>5 \%$ en postestrés & 18 \\
\hline & $\begin{array}{l}\text { Dilatación del ventrículo izquierdo } \\
\text { en reposo }\end{array}$ & 22 \\
\hline & $\begin{array}{l}\text { Dilatación del ventrículo izquierdo } \\
\text { en postestrés }\end{array}$ & 3 \\
\hline
\end{tabular}

FEVI: fracción de eyección del ventrículo izquierdo. 
presentaban defectos fijos con o sin componente reversible. Al evaluar la función sistólica, 29\% fue anormal; 22\% mostró FEVI disminuida en reposo y $23 \%$ en postestrés. De los casos con datos adecuados en control de calidad del gatillado electrocardiográfico, $18 \%$ tenían caída $>5 \%$ en postestrés. Con respecto a la dilatación ventricular, $22 \%$ la presentaban en reposo y $3 \%$ solo en postestrés.

$\mathrm{Al}$ analizar la mortalidad, $24 \%$ de las muertes fueron atribuidas a causas cardíacas y en $22 \%$ de los pacientes sin esta causa, fue atribuido a cáncer en el certificado de defunción. En la Tabla 3 se analizaron las causas de muerte por cualquier origen; en el análisis bivariado, se observó menor Hazard Ratio (HR) en mujeres que en hombres [HR: 0,56; IC: $0,43-0,72 ; p<0,001]$, mientras que aquellos pacientes con antecedente de EC conocida tuvieron mayor $\mathrm{HR}$ que los que no presentaban dicho antecedente [HR: 1,32; IC: $1,0-1,7 ; p=0,029$ ]. En el análisis multivariado, se encontró como factor protector de mortalidad de cualquier causa, al sexo femenino [HR: 0,52; IC 0,40-0,68; $<<0,001$ ] $\mathrm{y}$ como factor de riesgo, la edad mayor o igual de 46 años [HR: 2,0; IC: 1,53-2,62; $\mathrm{p}<0,001]$.

En la Tabla 4, al revisar los factores de riesgo de muerte por causa cardíaca, en el análisis bivariado, lo fueron el presentar un SPECT anormal [HR 3,74; IC: 2,15-6,51; p < 0,001], así como la presencia de isquemia [HR: 2,12; IC: 1,31-3,46; $\mathrm{p}=0,002]$ o infarto de miocardio [HR: 3,48; IC: 2,02-5,97; $\mathrm{p}<0,001]$. La FEVI, como variable continua, en reposo [HR: 0,95; IC: 0,94-0,96; $p<0,001$ ] y en postestrés [HR: 0,95; IC: 0,94-0,96; $\mathrm{p}<0,001]$ se asociaron positivamente a sobrevida. La dilatación del VI en las IPM tuvo un HR elevado [HR: 4,62; IC: 2,83-7,56; $\mathrm{p}<0,001$ ]. Por otra parte, en el análisis multivariado, una mejor FEVI postestrés también se asoció a mayor sobrevida [HR: 0,95; IC: 0,92-0,99; $\mathrm{p}=0,006$ ].

En las Figuras 1 y 2 se aprecian las curvas de mortalidad cardíaca; en la primera se comparan pacientes con SPECT normal versus anormal y en la segunda, casos sin isquemia versus con isquemia, observándose diferencias significativas en la sobrevida; los pacientes con estudio normal o sin isquemia tuvieron mejor tasa de sobrevida que los con SPECT alterado o con isquemia respectivamente [HR 3,74 y 2,12, respectivamente], siendo más significativa la diferencia al comparar IPM normal versus anormal (Figura 1).

Respecto a la seguridad de los estudios de perfusión miocárdica con DIP, no hubo reacciones adversas severas ni muertes durante las pruebas de estrés ni mientras se adquirían los SPECT ni en días posteriores. Para los efectos adversos menores se logró adecuado antagonismo con aminofilina e.v. de rutina y adicionalmente, en caso necesario.

Tabla 3. Causa de muerte global en pacientes sometidos a SPECT miocárdico con dipiridamol

\begin{tabular}{|c|c|c|c|c|}
\hline Análisis & Factor & Escala & HR (IC 95\%) & $\mathbf{p}$ \\
\hline \multirow[t]{7}{*}{ Bivariado } & \multirow[t]{2}{*}{ Sexo } & Hombre & 1 & - \\
\hline & & Mujer & $0,56(0,43 ; 0,72)$ & $<0,0001^{*}$ \\
\hline & \multirow[t]{3}{*}{ Edad } & Menor o igual a 45 años & 1 & - \\
\hline & & Entre 46 y 65 años & $1,28(0,47 ; 3,51)$ & 0,626 \\
\hline & & Mayor o igual a 66 años & $2,39(0,89 ; 6,45)$ & 0,084 \\
\hline & \multirow[t]{2}{*}{ EC conocida } & No & 1 & - \\
\hline & & Sí & $1,32(1,0 ; 1,7))$ & $0,029^{*}$ \\
\hline \multirow[t]{5}{*}{ Multivariado } & \multirow[t]{2}{*}{ Sexo } & Hombre & 1 & - \\
\hline & & Mujer & $0,52(0,40 ; 0,68)$ & $<0,001 *$ \\
\hline & \multirow[t]{3}{*}{ Edad } & Menor o igual a 45 años & 1 & - \\
\hline & & Entre 46 y 65 años & $2,0(1,53 ; 2,62)$ & $<0,001^{*}$ \\
\hline & & Mayor o igual a 66 años & - & - \\
\hline
\end{tabular}

HR: Hazard Ratio; IC: intervalo de confianza. *p significativa. EC: enfermedad coronaria. 
Tabla 4. Análisis de sobrevida con causa de muerte cardíaca en pacientes sometidos a SPECT miocárdico con dipiridamol

\begin{tabular}{|c|c|c|c|c|}
\hline & Factor & Escala & HR (IC 95\%) & $\mathbf{p}$ \\
\hline \multirow[t]{7}{*}{ Bivariado } & ECG estrés & $\begin{array}{l}\text { Negativo } \\
\text { Positivo }\end{array}$ & $\begin{array}{c}1 \\
1,02(0,5 ; 2,0)\end{array}$ & 0,966 \\
\hline & SPECT & $\begin{array}{l}\text { Normal } \\
\text { Anormal }\end{array}$ & $\begin{array}{c}1 \\
3,74(2,15 ; 6,51)\end{array}$ & $<0,001^{*}$ \\
\hline & Presencia y grado de isquemia miocárdica & $\begin{array}{l}\text { Ausente } \\
\text { Presente } \\
\text { Leve } \\
\text { Moderado } \\
\text { Severo }\end{array}$ & $\begin{array}{c}1 \\
2,12(1,31 ; 3,46) \\
1 \\
1,43(0,68 ; 3,01) \\
1,95(0,74 ; 5,13)\end{array}$ & $\begin{array}{c}0,002^{*} \\
- \\
0,343 \\
0,177\end{array}$ \\
\hline & Presencia y grado de infarto miocárdico & $\begin{array}{l}\text { Ausente } \\
\text { Presente } \\
\text { Leve } \\
\text { Moderado } \\
\text { Severo }\end{array}$ & $\begin{array}{c}1 \\
3,48(2,02-5,97) \\
1 \\
1,47(0,57 ; 3,79) \\
2,38(0,97 ; 5,83)\end{array}$ & $\begin{array}{c}<0,001 * \\
- \\
0,428 \\
0,059\end{array}$ \\
\hline & FEVI postestrés & Porcentual & $0,95(0,94 ; 0,96)$ & $<0,0001^{*}$ \\
\hline & FEVI reposo & Porcentual & $0,95(0,94 ; 0,96)$ & $<0,0001^{*}$ \\
\hline & Dilatación & $\begin{array}{l}\text { Ausente } \\
\text { Presente }\end{array}$ & $\begin{array}{c}1 \\
4,62(2,83 ; 7,56)\end{array}$ & $<0,0001^{*}$ \\
\hline Multivariado & FEVI postestrés & Porcentual & $0,95(0,92 ; 0,99)$ & $0,006^{*}$ \\
\hline
\end{tabular}

*p significativa. FEVI: fracción de eyección del ventrículo izquierdo. ECG: electrocardiograma. HR: Hazard Ratio; IC: intervalo de confianza.

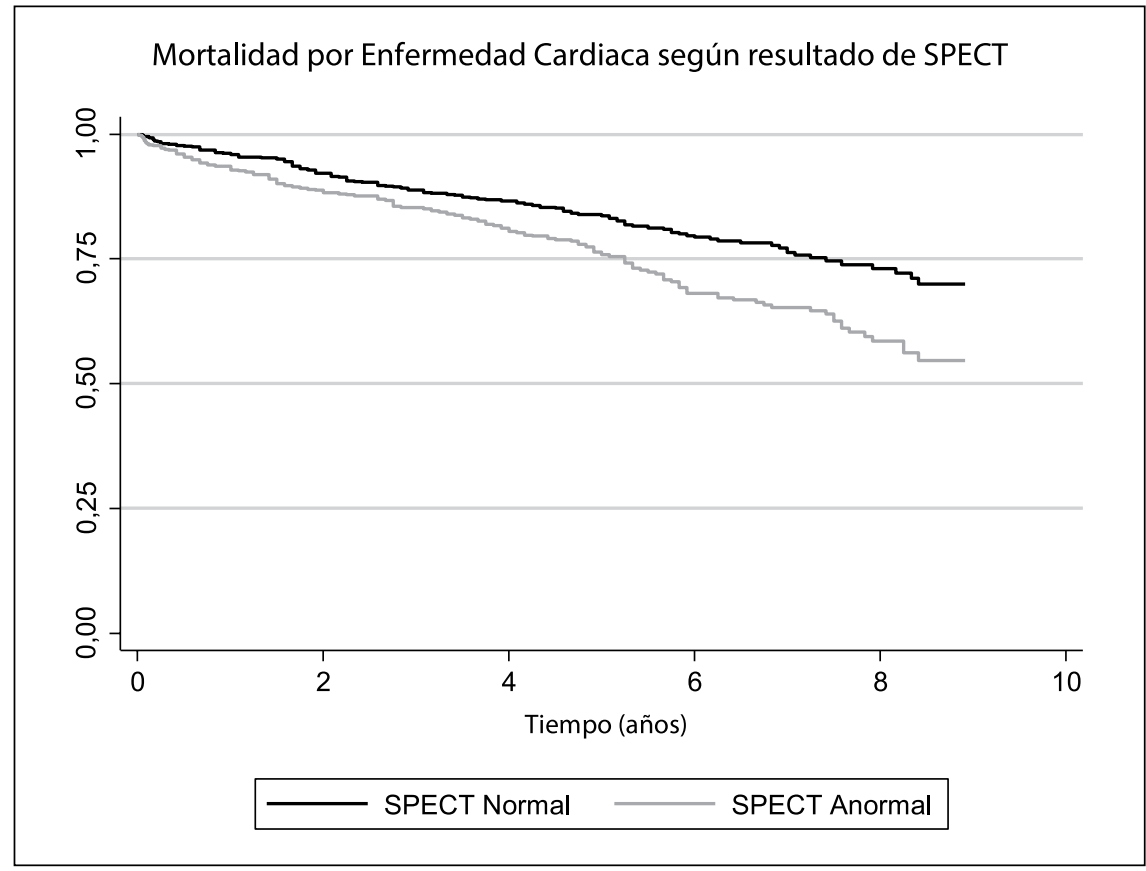

Figura 1. Curvas de sobrevida de Kaplan Meier: Mortalidad cardíaca según la presencia o ausencia de anormalidades en el SPECT gatillado $(p<0,001)$. 


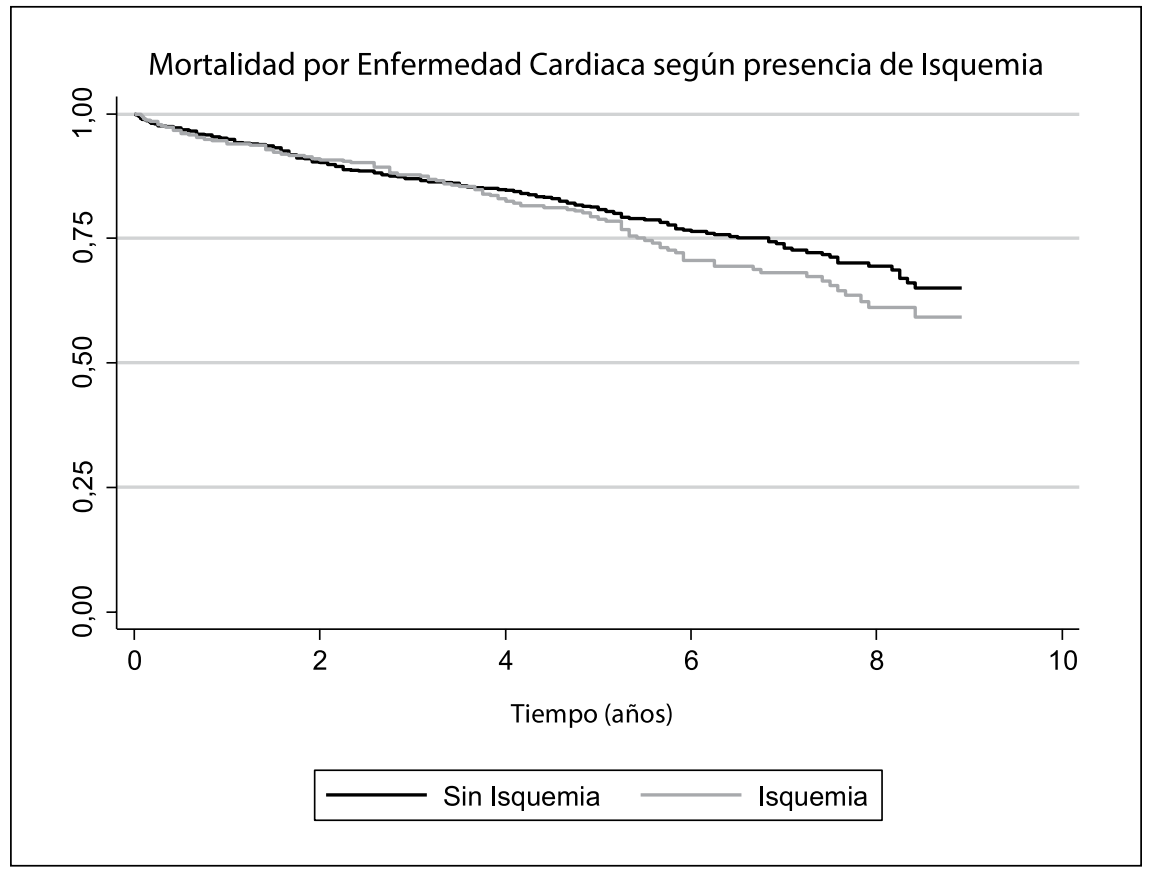

Figura 2. Curvas de sobrevida de Kaplan Meier: Mortalidad cardíaca según la presencia o ausencia de isquemia en el SPECT gatiIlado $(p=0,002)$.

\section{Discusión}

Las principales causas para realizar estudios con estrés farmacológico en evaluación de EC incluyen comorbilidades o historia de baja condición física; se deben utilizar vasodilatadores en presencia de bloqueo completo de rama izquierda o ritmo de marcapasos para evitar falsos positi$\operatorname{vos}^{13}$.

En las IPM, los parámetros del defecto de perfusión en cuanto a extensión se relacionan con la sobrevida ${ }^{19}$ y los obtenidos durante la prueba con vasodilatadores proveen similar valor pronóstico que el obtenido con ejercicio ${ }^{7}$. El estrés con ejercicio es preferido en pacientes capaces de lograr al menos 5 equivalentes metabólicos (METS) y alcanzar $85 \%$ de la frecuencia cardíaca máxima teórica para la edad (FCMT), ya que los efectos fisiológicos pueden ser observados directamente ${ }^{20}$, se aprovechan los datos aportados por la prueba de esfuerzo; también esta es predictora independiente de sobrevida, especialmente al alcanzar 10 o más METS $^{21}$. En un gran estudio de ecocardiografía con estrés físico ${ }^{22}$, se encontraron tasas de mortalidad cardíaca anuales de $2,26 \%$ y $0,84 \%$ en pacientes con y sin isquemia, con tasas de eventos cardíacos mayores en el grupo con isquemia de $0,84 \%$ versus $0,32 \%$ en los sin esta, en los que alcanzaron 10 METS y respuesta máxima ( $>85 \%$ de su FCMT). En una pequeña experiencia nuestra con $121 \mathrm{pa}-$ cientes en evaluación de EC capaces de alcanzar 10 METS se encontró isquemia en $24,8 \%$ y baja tasa de eventos cardiovasculares durante seguimiento promedio de 33 meses $^{23}$.

Se ha observado un incremento en el último tiempo del uso de estrés farmacológico comparado con el ejercicio en muchas regiones del mundo, con más de la mitad de los estudios realizados con vasodilatadores en la actualidad en nuestro medio $^{8}$. La mayoría de los pacientes referidos para estudios con vasodilatadores presentan probabilidad pretest moderada a alta de tener una EC, así como menor capacidad funcional y de 2 a 3 veces mayores tasas de eventos comparados con los pacientes sometidos a estrés de ejercicio ${ }^{24}$.

En nuestra revisión de casi 1.000 casos de adultos, un tercio de los pacientes presentaba antecedente de EC conocida, lo cual es similar a lo descrito en un estudio previo, en el cual 32\% de los pacientes bajo efecto de vasodilatador tenía infarto al miocardio ${ }^{25}$; sin embargo, en ese trabajo, el promedio de edad era ligeramente superior 
(70,4 $\pm 11,3$ años versus $66 \pm 11$ años del nuestro), lo que pudiera explicar mayor enfermedad o sesgo de referencia.

En este estudio con vasodilatadores, los principales factores de riesgo de mortalidad de cualquier causa fueron el antecedente de EC conocida, el sexo masculino y la edad mayor o igual a 46 años. En otro trabajo a mayor plazo y de sobre 12.000 pacientes evaluados con SPECT en ejercicio normal, entre los factores de riesgo de mortalidad se demostró a la edad y al sexo masculino, así como la capacidad funcional ${ }^{25}$. El 44\% de nuestras IPM presentaban alguna alteración (77\% isquemia y $84 \%$ disfunción sistólica), siendo esos hallazgos los principales predictores de mortalidad y eventos cardíacos $^{8}$. Otro estudio similar mostró aumento de las tasas de eventos cardiacos anuales de forma independiente tanto para casos con alteraciones de perfusión, como aquellos con FEVI disminui$\mathrm{da}$, con tasas de $5,1 \%$ y $7,4 \%$, respectivamente ${ }^{27}$. Nosotros no encontramos diferencia en el grado de extensión de la isquemia pero sí del infarto de miocardio, para el análisis de sobrevida.

También se apreciaron diferencias en la sobrevida de los pacientes, ya que al encontrarse alguna anormalidad en el SPECT, o presencia de isquemia, se aumenta la mortalidad de causa cardíaca durante el seguimiento, lo que es concordante con estudios previos. En un metaanálisis se observó que en sujetos sometidos a estrés farmacológico, con uno o más defectos de perfusión en el SPECT, la tasa sumada anualizada para muerte cardíaca e infarto al miocardio fue $9,98 \%$ versus $1,78 \%$ en aquellos con imágenes normales ${ }^{24}$.

La seguridad del DIP intravenoso como vasodilatador coronario con SPECT fue demostrada en un importante trabajo multicéntrico de casi 74.000 casos, que presentó riesgo muy bajo de efectos cardiovasculares adversos serios, similar a lo observado en la prueba con ejercicio ${ }^{28}$. Se ha publicado el uso seguro de esta droga en diversos tipos de pacientes frágiles, adultos mayores, diabéticos, hipertensos, obesos, con insuficiencia renal crónica, postinfarto agudo al miocardio recien$\mathrm{te}^{7,28-35}$. Sin embargo, se plantea tener cierta cautela en accidentes vasculares cerebrales recientes; en un trabajo de sobre 400 casos, solo se publicó un episodio de tipo isquémico transitorio ${ }^{30}$. El uso de DIP es adecuado en niños, en evaluación de enfermedad de Kawasaki y cardiopatías congéni$\operatorname{tas}^{36}$. Debe recordarse que está contraindicado en asma bronquial severa ${ }^{18}$. En nuestro trabajo, no se observaron efectos adversos graves durante su utilización; similar a lo observado con dosis altas $(0,84 \mathrm{mg} / \mathrm{kg})^{35}$.

Una fortaleza de esta investigación es que la muestra seleccionada representa la realidad de la práctica cotidiana, con gran número de pacientes consecutivos con reportes originales. Una debilidad fue el diseño retrospectivo, que limitó el tipo de datos obtenidos y la ausencia de seguimiento clínico, obteniendo solo los datos de causa muerte del registro nacional de defunción, no valorándose otros eventos cardíacos mayores, como infarto de miocardio o menores como requerimiento de revascularización coronaria.

\section{Conclusión}

La presencia de anormalidades en el SPECT miocárdico como isquemia, infarto, dilatación o disfunción sistólica izquierda especialmente postestrés, fueron predictores independientes de mortalidad cardíaca en la población sometida a DIP como agente estresor, en análisis de Cox.

Agradecimientos: Al señor Ariel Castro Lara Q.F. de la Oficina de Apoyo a la Investigación Clínica del Hospital Clínico, por su ayuda en el análisis estadístico del trabajo.

\section{Referencias}

1. Fihn SD, Gardin JM, Abrams J, Berra K, Blankenship JC, Dallas AP, et al. 2012 ACCF/AHA/ACP/AATS/PCNA/ SCAI/STS Guideline for the diagnosis and management of patients with stable ischemic heart disease: a report of the American College of Cardiology Foundation/ American Heart Association Task Force on Practice Guidelines, and the American College of Physicians, American Association for Thoracic Surgery, Preventive Cardiovascular Nurses Association, Society for Cardiovascular Angiography and Interventions, and Society of Thoracic Surgeons. J Am Coll Cardiol 2012; 60 (24): e44-e164.

2. Ministerio de Salud. Departamento de Epidemiología. Unidad de Estudios y VENT. Cálculos en base a datos del Departamento de Estadísticas e Información en Salud, Estadísticas Vitales MINSAL [Internet]. 2009. Available from: http://epi.minsal.cl/infarto-agudo-al-miocardio-situacion-epidemiologica/ 
3. Massardo T, Alarcon L, Spuler J. Risk stratification of coronary artery disease using radionuclides. Current status of clinical practice. Rev Esp Med Nucl Imagen Mol 2017; 36 (6): 377-87.

4. Rozanski A, Berman DS. The efficacy of cardiovascular nuclear medicine exercise studies. Semin Nucl Med 1987; 17 (2): 104-20.

5. Verberne HJ, Acampa W, Anagnostopoulos C, Ballinger J, Bengel F, De Bondt P, et al. EANM procedural guidelines for radionuclide myocardial perfusion imaging with SPECT and SPECT/CT: 2015 revision. Eur J Nucl Med Mol Imaging 2015; 42 (12): 1929-40.

6. Iskandrian AS, Verani MS, Heo J. Pharmacologic stress testing: mechanism of action, hemodynamic responses, and results in detection of coronary artery disease. J Nucl Cardiol 1994; 1 (1): 94-111.

7. Henzlova MJ, Duvall WL, Einstein AJ, Travin MI, Verberne HJ. ASNC imaging guidelines for SPECT nuclear cardiology procedures: Stress, protocols, and tracers. J Nucl Cardiol 2016; 23 (3): 606-39.

8. Bajaj NS, Singh S, Farag A, El-Hajj S, Heo J, Iskandrian $\mathrm{AE}$, et al. The prognostic value of non-perfusion variables obtained during vasodilator stress myocardial perfusion imaging. J Nucl Cardiol 2016; 23 (3): 390413.

9. Takx RA, Blomberg BA, El Aidi H, Habets J, de Jong PA, Nagel E, et al. Diagnostic accuracy of stress myocardial perfusion imaging compared to invasive coronary angiography with fractional flow reserve meta-analysis. Circ Cardiovasc Imaging 2015; 8 (1).

10. Danad I, Szymonifka J, Twisk JWR, Norgaard BL, Zarins CK, Knaapen P, et al. Diagnostic performance of cardiac imaging methods to diagnose ischaemia-causing coronary artery disease when directly compared with fractional flow reserve as a reference standard: a meta-analysis. Eur Heart J 2017; 38 (13): 991-8.

11. Wolk MJ, Bailey SR, Doherty JU, Douglas PS, Hendel RC, Kramer CM, et al. ACCF/AHA/ASE/ASNC/HFSA/ HRS/SCAI/SCCT/SCMR/STS 2013 multimodality appropriate use criteria for the detection and risk assessment of stable ischemic heart disease: a report of the American College of Cardiology Foundation Appropriate Use Criteria Task Force, American Heart Association, American Society of Echocardiography, American Society of Nuclear Cardiology, Heart Failure Society of America, Heart Rhythm Society, Society for Cardiovascular Angiography and Interventions, Society of Cardiovascular Computed Tomography, Society for Cardiovascular Magnetic Resonance, and Society of Thoracic Surgeons. J Am Coll Cardiol 2014; 63 (4): 380-406.
12. Hendel RC, Berman DS, Di Carli MF, Heidenreich PA, Henkin RE, Pellikka PA, et al. ACCF/ASNC/ACR/AHA/ ASE/SCCT/SCMR/SNM 2009 appropriate use criteria for cardiac radionuclide imaging: a report of the American College of Cardiology Foundation Appropriate Use Criteria Task Force, the American Society of Nuclear Cardiology, the American College of Radiology, the American Heart Association, the American Society of Echocardiography, the Society of Cardiovascular Computed Tomography, the Society for Cardiovascular Magnetic Resonance, and the Society of Nuclear Medicine. Circulation 2009; 119 (22): e561-87.

13. Alexanderson EBN, Bouyoucef S-E, Dondi M, Dorbala S, Einstein AJ, El-Haj N, et al. Nuclear Cardiology: Guidance on the implementation of SPECT Myocardial Perfusion Imaging. . IAEA HUMAN HEALTH SERIES 2016; 23 (Rev. 1): 101.

14. Morales DCV, Bhavnani SP, Ahlberg AW, Pullatt RC, Katten DM, Polk DM, et al. Coronary risk equivalence of diabetes assessed by SPECT-MPI. J Nucl Cardiol 2017.

15. Rozanski A, Gransar H, Hayes SW, Friedman JD, Hachamovitch R, Berman DS. Comparison of long-term mortality risk following normal exercise vs adenosine myocardial perfusion SPECT. J Nucl Cardiol 2010; 17 (6): 999-1008.

16. Uretsky S, Rozanski A. Long-term outcomes following a normal stress myocardial perfusion scan. J Nucl Cardiol 2013; 20 (5): 715-8.

17. Ottenhof MJ, Wai MC, Boiten HJ, Korbee RS, Valkema R, van Domburg RT, et al. 12-Year outcome after normal myocardial perfusion SPECT in patients with known coronary artery disease. J Nucl Cardiol 2013; 20 (5): 748-54.

18. Massardo T, Jaimovich R, Canessa J, Castro G, Soto JR. Actualización de protocolos de cardiología nuclear para evaluación y manejo de enfermedad coronaria. Sociedad Chilena de Cardiología y Cirugía Cardiovascular. Rev Chil Cardiol 2010; 29: 13.

19. Hachamovitch R, Hayes SW, Friedman JD, Cohen I, Berman DS. Comparison of the short-term survival benefit associated with revascularization compared with medical therapy in patients with no prior coronary artery disease undergoing stress myocardial perfusion single photon emission computed tomography. Circulation 2003; 107 (23): 2900-7.

20. Mangla A, Oliveros E, Williams KA, Sr., Kalra DK. Cardiac Imaging in the Diagnosis of Coronary Artery Disease. Curr Probl Cardiol 2017; 42 (10): 316-66.

21. Bourque JM, Charlton GT, Holland BH, Belyea CM, Watson DD, Beller GA. Prognosis in patients achieving 
$>/=10$ METS on exercise stress testing: was SPECT imaging useful? J Nucl Cardiol 2011; 18 (2): 230-7.

22. Peteiro J, Bouzas-Mosquera A, Broullon F, Martínez D, Yanez J, Castro-Beiras A. Value of an exercise workload $>/=10$ metabolic equivalents for predicting inducible myocardial ischemia. Circ Cardiovasc Imaging 2013; 6 (6): 899-907.

23. González J, Prat H, Swett E, Berrocal I, Fernández R, Zhindon JP, et al. [Follow-up of patients with good exercise capacity in stress test with myocardial single-photon emission computed tomography (SPECT)]. Rev Med Chile 2015; 143 (11): 1426-34.

24. Navare SM, Mather JF, Shaw LJ, Fowler MS, Heller GV. Comparison of risk stratification with pharmacologic and exercise stress myocardial perfusion imaging: a meta-analysis. J Nucl Cardiol 2004; 11 (5): 551-61.

25. Hachamovitch R, Berman DS, Shaw LJ, Kiat H, Cohen I, Cabico JA, et al. Incremental prognostic value of myocardial perfusion single photon emission computed tomography for the prediction of cardiac death: differential stratification for risk of cardiac death and myocardial infarction. Circulation 1998; 97 (6): 53543.

26. Rozanski A, Gransar H, Min JK, Hayes SW, Friedman JD, Thomson LE, et al. Long-term mortality following normal exercise myocardial perfusion SPECT according to coronary disease risk factors. J Nucl Cardiol 2014; 21 (2): 341-50.

27. Travin MI, Heller GV, Johnson LL, Katten D, Ahlberg AW, Isasi CR, et al. The prognostic value of ECG-gated SPECT imaging in patients undergoing stress Tc-99m sestamibi myocardial perfusion imaging. J Nucl Cardiol 2004; 11 (3): 253-62.

28. Lette J, Tatum JL, Fraser S, Miller DD, Waters DD, Heller G, et al. Safety of dipyridamole testing in 73,806 patients: the Multicenter Dipyridamole Safety Study. J
Nucl Cardiol 1995; 2 (1): 3-17.

29. Aksut SV, Port S, Collier BD, Hoffmann RG, Massardo T, Hellman RS, et al. Dipyridamole thallium-201 myocardial imaging. Complications associated with oral and intravenous routes of administration. Clin Nucl Med 1988; 13 (11): 786-8.

30. Lette J, Carini G, Tatum JL, Paquet N, Bisson G, Picard $\mathrm{M}$, et al. Safety of dipyridamole testing in patients with cerebrovascular disease. Am J Cardiol 1995; 75 (7): 535-7.

31. Gerson MC, Moore EN, Ellis K. Systemic effects and safety of intravenous dipyridamole in elderly patients with suspected coronary artery disease. Am J Cardiol 1987; 60 (16): 1399-401.

32. Cortigiani L, Zanetti L, Bigi R, Desideri A, Fiorentini C, Nannini E. Safety and feasibility of dobutamine and dipyridamole stress echocardiography in hypertensive patients. J Hypertens 2002; 20 (7): 1423-9.

33. Heller GV, Brown KA, Landin RJ, Haber SB. Safety of early intravenous dipyridamole technetium $99 \mathrm{~m}$ sestamibi SPECT myocardial perfusion imaging after uncomplicated first myocardial infarction. Early Post MI IV Dipyridamole Study (EPIDS). Am Heart J 1997; 134 (1): 105-11.

34. Ando S, Ashihara T, Ando H, Mitsuoka W, Tagawa H, Fukuoka T, et al. Safety and accuracy of dipyridamole thallium myocardial scintigraphy in elderly patients. Jpn Heart J 1993; 34 (3): 245-54.

35. Cortigiani L, Desideri A, Gigli G, Vallebona A, Terlizzi $\mathrm{R}$, Giusti R, et al. Clinical, resting echo and dipyridamole stress echocardiography findings for the screening of renal transplant candidates. Int J Cardiol 2005; 103 (2): 168-74.

36. Massardo T, Coll C, Prat H, Miranda K, Gutiérrez D, Arenas A, Alay R. Experiencia en estudios de SPECT de perfusión miocárdica en población pediátrica. Alasbimn Journal 7 (26): October 2004 Article N AJ26-3. 2004. 